Journal of Computer Science 3 (5): 353-360, 2007

ISSN 1549-3636

(C) 2007 Science Publications

\title{
A Novel Image Component Transmission Approach to Improve Image Quality and Energy Efficiency in Wireless Sensor Networks
}

\author{
Wei Wang, Dongming Peng, Honggang Wang, Hamid Sharif \\ Department of Computer and Electronics Engineering, University of Nebraska-Lincoln, USA
}

\begin{abstract}
Due to resource constraints, digital image transmissions are a significant challenge for image sensor based Wireless Sensor Networks (WSN). In this paper, we propose a novel robust and energy-efficient scheme, called Image Component Transmission (ICT) in WSN by providing various levels of reliability during image transmissions. Different components in the embedded wavelet image bit stream are considered and essential components of the compressed image such as the information for the positions of significant wavelet coefficients are transmitted with higher quality assurance. Relatively less important components such as the information for the values of pixels are transmitted with lower overhead. Simulation results show the proposed scheme achieves higher energy efficiency in WSN, with image transmission quality enhanced considerably by up to $6 \mathrm{~dB}$ in terms of the Peak Signal to Noise Ratio (PSNR).
\end{abstract}

Keywords: Image Component Transmission, Wireless Sensor Network, Discrete Wavelet Transform.

\section{INTRODUCTION}

Recent advances in image processing, Radio Frequency (RF) and micro-electronic technologies have brought enormous development of image transmission over Wireless Sensor Networks (WSN) ${ }^{[1]}$. Unlike usual image transmission designs in wireless environment, energy efficiency has the highest priority in WSN. Usually, image data is bulk sized and contains a lot of redundancy ${ }^{[2]}$, which leads to challenges in designing energy efficient image transmission schemes over WSN. Wavelet based image compression techniques such as zerotree coding ${ }^{[3-4]}$ and EBCOT ${ }^{[5]}$ achieve high compression ratio and are embedded multirate in nature, providing unequal importance attributes. Unequal importance here means different parts of the compressed bit streams that exhibit different perceptual and structural importance ${ }^{[2]}$. Important parts provide critical information for reconstruction of the original image, whereas other parts may provide fine information in higher resolution ${ }^{[6]}$.

The information of natural digital image is generally conveyed by the positions of image pixels of various values. There are different fault-tolerance levels for disturbance on the compressed image bits to be transmitted in wireless networks, particularly wireless sensor network environment. Wavelet based image compression produces shape and position information of the regions (e.g. how small magnitude coefficients are clustered), as well as the lighting information in regions (e.g., how large magnitude coefficients are valued). Transmission errors in shape and position information lead to high difficulties for reconstructing the original image, while errors in the magnitudes are more tolerable during transmission.

Our approach can be expressed in two aspects, i.e., (1) Identification of the essential and important components, and (2) Reliable and efficient transmission of important parts. In the first step, wavelet coefficients are produced after Discrete Wavelet Transform (DWT) on the original image as in the main-stream compression algorithms, and embedded bitstreams are produced after compression. In the embedded bitstreams, multiple important parts are identified and marked associated with their importance levels. In the second step, unequally important transmissions are applied to different components in the compressed image bit stream. More reliable transmission of the important parts enhances image quality, while less effort is put on unimportant parts, leading to energy efficiency. Overall speaking, by exploring unequal importance attribute of compressed image bits, our

Corresponding Author: Wei Wang, Department of Computer and Electronics Engineering, University of Nebraska Lincoln at Omaha Campus,Room 313A, Peter Kiewit Institute1110 South 67th Street, Omaha, NE 68182 , USA 
proposed Image Component Transmission (ICT) approach achieves high energy efficiency while enhancing image transmission quality in WSN.

Some of the previous researches have been performed to explore the unequal importance image transmission over WSN for energy efficiency. However, most of them focus on the multi resolution levels and the embedded progressive characteristics of wavelet based image compression. How to energy-efficiently transmit image over WSN by exploring unequal importance nature of position information (i.e. pixel structure information) and value information (i.e. pixel magnitude information) is not extensively discussed in literature.

The research in ${ }^{[2]}$ proposes a joint source channel coding approach for energy efficient JPEG 2000 image transmission in WSN, by applying different error resilient coding protection to different levels or layers of bitstreams. This approach extensively explores the multi resolution nature of bitstreams; however, the unequal importance between structure information and magnitude information is not fully identified. In [9] multiple bitstreams are created to constrain error propagation in a sub-tree, and hierarchical unequal error protections are applied. Again, this approach is still image-layer (or level, hierarchy) oriented, without considering the unequal importance between the structure information and magnitude information. Researches in ${ }^{[1]}$ and ${ }^{[10]}$ investigate the tradeoff between energy consumption and image quality. By balancing computational energy consumption and transmission energy through image compression, energy efficient transmission in WSN is achieved. Those approaches apply uniform protection to the whole image, and do not look into the difference between the structure information and magnitude information.

To the best of our knowledge, the unequal importance nature of compressed image bits, in terms of the structure (i.e., pixel position, shape) and the magnitude (i.e., pixel value) information, is not fully studied in state-of-the-art image transmission schemes over wireless networks, particularly WSN where resources (e.g., energy costs, channel qualities, etc. ) are severely constrained. On the other hand, such unequal importance would provide extraordinary opportunities for energy-efficient and robust image transmission schemes over WSN. In this paper, the proposed ICT scheme takes this novel view and synthesizes it with WSN design in a cross-layer panorama, which has the major difference from previous works.
Energy Consumption of Multirate Image Component Transmission: There have been some researches on supporting and interacting multirate signal processing applications in the WSN design. In our previous work on the multirate signal processing platform over WSN ${ }^{[7-8]}$, the average energy consumption of transmitting and receiving a data packet can be expressed in Equation (1).

$$
\begin{aligned}
& E_{p k t}=E^{S U C C} \cdot p^{S U C C}+E_{R T S}^{\text {Fail }} \cdot p_{R T S}^{\text {Fail }} \\
& +E_{C T S}^{\text {Fail }} \cdot p_{C T S}^{\text {Fail }}+E_{D A T A}^{\text {Fail }} \cdot p_{D A T A}^{\text {Fail }}+E_{A C K}^{\text {Fail }} \cdot p_{A C K}^{\text {Fail }} \\
& +p^{S U C C} \cdot E_{D A T A_{-} R}^{\text {SUCC }}+p_{R T S}^{\text {fail }} \cdot E_{R T S_{-} R}^{\text {fail }}+p_{C T S}^{\text {fail }} \cdot E_{C T S_{-} R}^{\text {fail }} \\
& +p_{D A T A}^{\text {fail }} \cdot E_{D A T A_{-} R}^{\text {fail }}+p_{A C K}^{\text {fail }} \cdot E_{A C K_{-} R}^{\text {fail }}
\end{aligned}
$$

The probabilities and energy consumptions for RTS, CTS, DATA, ACK packet transmissions, failed or successful, are expressed below. BER is the desirable bit error ratio; $L_{R T S}$ is the RTS packet length; $p$ is the probability of a single node sending packet, $N$ is the neighbor count of that node; $P^{T X}$ and $P^{R X}$ denote the power required for transmitting or receiving, respectively. $T_{R T S}$ is the RTS transmission time, and $T_{\text {CTS_timeout }}$ denotes the time out value of receiving CTS packet. Detailed descriptions can be referenced in [8].

$$
\begin{aligned}
& p_{R T S}^{\text {Fail }}=1-(1-B E R)^{L_{R T S}} \cdot p \cdot(1-p)^{N} \\
& E_{R T S}^{\text {Fail }}=P^{T X} \cdot T_{R T S}+P^{R X} \cdot T_{C T S \_ \text {timeout }}
\end{aligned}
$$

The probability of CTS, DATA and ACK packet failure, and the corresponding energy consumption can be expressed in Equations (4) - (5):

$$
\begin{aligned}
& p_{C T S}^{\text {Fail }}=\left(1-p_{R T S}^{\text {Fail }}\right) \cdot\left(1-(1-B E R)^{L_{C T S}}\right) \\
& E_{C T S}^{\text {Fail }}=P^{T X} \cdot T_{R T S}+P^{R X} \cdot T_{C_{T S} \text { timeout }}
\end{aligned}
$$

The packet error rate due to DATA packet failure, and the corresponding energy consumption can be expressed in Equations (6) - (7):

$$
\begin{aligned}
& p_{D A T A}^{\text {Fail }}=\left(1-p_{R T S}^{\text {Fail }}\right) \cdot\left(1-p_{C T S}^{\text {Fail }}\right) \cdot\left(1-(1-B E R)^{L_{D A T A}}\right) \\
& E_{D A T A}^{\text {Fail }}=P^{T X} \cdot T_{R T S}+P^{R X} \cdot T_{C T S}+P^{T X} \cdot T_{D A T A}+P^{R X} \cdot T_{A C K}
\end{aligned}
$$

Similarly, the packet error rate due to ACK packet failure, and the corresponding energy consumption can be expressed in Equations (8) - (9): 
$p_{A C K}^{F a i l}=\left(1-p_{R T S}^{F a i l}\right) \cdot\left(1-p_{C T S}^{\text {Fail }}\right) \cdot\left(1-p_{D A T A}^{\text {Fail }}\right) \cdot\left(1-(1-B E R)^{L_{A C K}}\right)$

$E_{A C K}^{F a i l}=P^{T X} \cdot T_{R T S}+P^{R X} \cdot T_{C T S}+P^{T X} \cdot T_{D A T A}+P^{R X} \cdot T_{A C K_{-} \text {timeou }}$

Above analysis gives the energy penalty and corresponding probability of packet loss events. The probability of successfully transmission of a packet, and the corresponding energy consumption can be expressed in Equations (10) - (11):

$$
\begin{aligned}
& p^{S U C C}=\left(1-p_{R T S}^{F a i l}\right) \cdot\left(1-p_{C T S}^{F a i l}\right) \cdot\left(1-p_{D A T A}^{F a i l}\right) \cdot\left(1-p_{A C K}^{F a i l}\right) \\
& E^{S U C C}=P_{R T S}^{T X} \cdot T_{R T S}+P^{R X} \cdot T_{C T S}+P_{D A T A}^{T X} \cdot T_{D A T A}+P^{R X} \cdot T_{A C K}
\end{aligned}
$$

Similarly, the statistical energy consumption of receiving a packet can be expressed in Equation (12)(15):

$$
\begin{aligned}
& E_{R T S \_R}^{\text {fail }}=P^{R X} \cdot T_{R T S} \text { timeout } \\
& E_{C T S \_R}^{f a i l}=P^{R X} \cdot T_{R T S}+P_{C T S}^{T X} \cdot T_{C T S}+P^{R X} \cdot T_{\text {DATA_timeout }} \\
& E_{D A T A R}^{f a i l}=P^{R X} \cdot T_{R T S}+P_{C T S}^{T X} \cdot T_{C T S}+P^{R X} \cdot T_{D A T A} \\
& E_{A C K_{-} R}^{f a i l}=P^{R X} \cdot T_{R T S}+P_{C T S}^{T X} \cdot T_{C T S}+P^{R X} \cdot T_{D A T A}+P_{A C K}^{T X} \cdot T_{A C K}
\end{aligned}
$$

The above equations (1)-(15) give the expression of packet level energy consumption, and the parameters in Equation (1) can be expressed by Equations (2)-(15). Furthermore, Equation (2)-(15) can be expressed as functions of desirable BER. In Equation (3), (5), (7), (9), (11), (13), (14), and (15) the transmission power can be expressed in terms of the desirable BER. According to our research published in ${ }^{[7-8]}$, the desirable transmission power can be determined by the BER requirement, channel attenuation, modulation schemes and transmission rate. For BPSK, QPSK and QAM modulation schemes the relationship between desirable transmission power and desirable BER is shown in Equations (16-17):

$$
\begin{aligned}
& P s^{B P S K, Q P S K}=R s \cdot b \cdot\left[\operatorname{erfc}^{-1}(2 \cdot B E R)\right]^{2} \cdot \frac{N_{0}}{A} \\
& P s^{M-Q A M}=\frac{1}{3} \cdot R s \cdot b \cdot\left(b^{2}-1\right) \cdot\left[\operatorname{erfc} c^{-1}\left(\frac{1}{2} \cdot\left(1-\frac{1}{b}\right)^{-1} \cdot B E R\right)\right]^{2} \cdot \frac{N_{0}}{A}(17)
\end{aligned}
$$

From above analysis on packet level energy consumption in Equations (1)-(17), we can observe that the energy consumption of transmitting and receiving a packet can be taken as a function of desirable BER. Given the factors of packet length (LDATA), overhead packet length (LRTS, LCTS, LACK), packet transmission time (TRTs, Tcts, TDATA, TACK, which can be solved by providing transmission data rate and the corresponding packet length), collision condition ( $\mathrm{p}$ and $\mathrm{N})$, receiving power, and channel conditions (N0 and A), Equation (1) can be rewritten in a simpler form, i.e., an explicit function of desirable BER:

$$
E_{p k t}=f(B E R)
$$

The relationship between packet level energy consumption and BER in Equation (18) is monotonous because all the factors in Equation (1) are monotonously increasing when BER increases. Once expressed with packet level energy consumption, the whole energy consumption of transmitting and receiving each image can be modeled in a componentbased scheme.

For ICT, the compressed image is segmented into several components with unequal importance in terms of the structure information and magnitude information. According to packet level energy consumption shown in Equation (1), we can get component level energy consumption expressed in Equation (19) and (20). Equation (19) denotes the energy consumption of important component containing structure information, and Equation (20) denotes the energy consumption of unimportant component containing magnitude information. In Equation (19), Hip denotes the size of the component containing structure information at level i, Lip denotes the transmission frame length for that component. Epkt is BER related parameters. Here BERip is the desirable BER requirement for component containing structure information in level $\mathrm{i}$. BERiv is for component containing magnitude information.

$$
\begin{aligned}
& E_{i p}=\frac{H_{i p}}{L_{i p}} \cdot E_{p k t}\left(B R_{i p}\right) \\
& E_{i v}=\frac{H_{i v}}{L_{i v}} \cdot E_{p k t}\left(B E R_{i v}\right)
\end{aligned}
$$

By summing up all the energy consumed by each component at each level of compression, the total energy consumption of N-level bitstream transmission can be expressed in Equation (21).

$$
E=\sum_{i=0}^{N-1} \frac{H_{i, j}}{L_{i, j}} \cdot E_{p k t}\left(B E R_{i, j}\right)
$$

where $j \in\{p, v\}$, denoting whether this component containing structure information (i.e. position) or magnitude (i.e., value) information.

Equation (21) gives the close form expression of energy consumption for image component transmission. Again, the total energy consumption for component based image transmission is solely determined by desirable BER requirements of each component, as long as given the factors of component size, transmission frame size, and the transmission rate. In addition, if the 
desirable BER is reduced for some components, then total energy consumption is reduced due to the monotonic function of energy consumption and desirable BER requirement for each component.

One contribution of ICT is that it provides flexible mechanisms to design BER distribution vector $\mathrm{B}=\left[\mathrm{BER}_{0 \mathrm{p}}, \mathrm{BER}_{0 \mathrm{v}}, \mathrm{BER}_{1 \mathrm{p}}, \mathrm{BER}_{1 \mathrm{v}},, \ldots \ldots\right.$ BER(n-1)p, $B E R(n-1) v]$ (each element corresponding to the desirable BER requirement assigned to the component)], in order to achieve robustness and energy efficiency. This approach takes advantage of the inherent inequality of image data importance within compressed bitstreams. By scaling down BER requirement of unimportant components, energy consumption is significantly reduced without perceptible image quality loss. On the other hand, for transmission of an image with the same energy consumption, using component approach has better image quality, because more effort is put on important components to assure image quality, while less effort is put on unimportant part to achieve energy saving.

\section{Proposed ICT Approach: Essential Component} Identification And P-V Based Unequal Error Protection (UEP): Wavelet based compression algorithms generally output the structural information in addition to the pixel magnitude coefficients ${ }_{[6]}$. For example, the SPIHT algorithm in ${ }^{[4]}$ encodes the data sets that correspond to trees of coefficients. Although we take tree based compression algorithms as examples in this paper, the proposed image component transmission methodology is generic and independent of specific wavelet image compression algorithms. It can be easily extended to other wavelet based image compression algorithms, because essential components are inheritance of the original image itself. As long as the image compression algorithms can produce unequal importance of their output streams consisting of pixel position (e.g., how small wavelet coefficients are clustered and distributed) and value information (e.g., how large wavelet coefficients are valued) separately, they can always be applied to the proposed ICT framework.

Figure 1 shows the wavelet coefficients and trees of wavelet coefficients after DWT is applied to the original image.

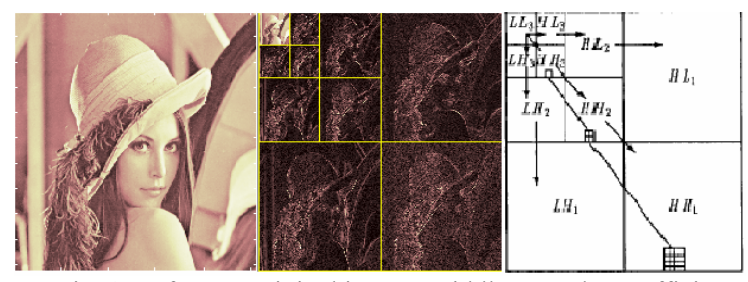

Fig. 1: Leftmost: original image; Middle: wavelet coefficients; Rightmost: tree structure of wavelet coefficients
The embedded output bitstreams after compression is shown in Figure 2, starting with rough image (i.e., coarsest resolution) followed by interleaving of tree structure information and magnitude information at various resolution levels. For example, the H0p symbols denote information bits of tree structure in the highest wavelet coefficient level, while the H1v symbols denote the information bits of magnitudes in the second highest level. In the proposed approach, all the tree structure information bits Hip $(i=0,1, \ldots n)$ are separated with different importance levels after the component identification process. Unequal importance is applied to structure information and magnitude information in different levels of wavelet coefficients, leading to the unequal importance of transmission that will be described in next subsection.

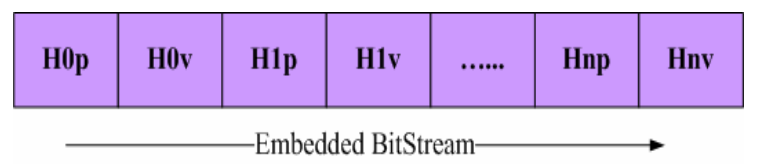

Fig. 2: Embedded output streams of interleaving tree structure information (p) and magnitude information (v).

Essential components can be identified from compressed embedded bitstreams. In this subsection, we discuss how to energy-efficiently and robustly transmit those bitstreams in an unequal importance protection approach. Figure 3 shows the data flow of such ICT framework.

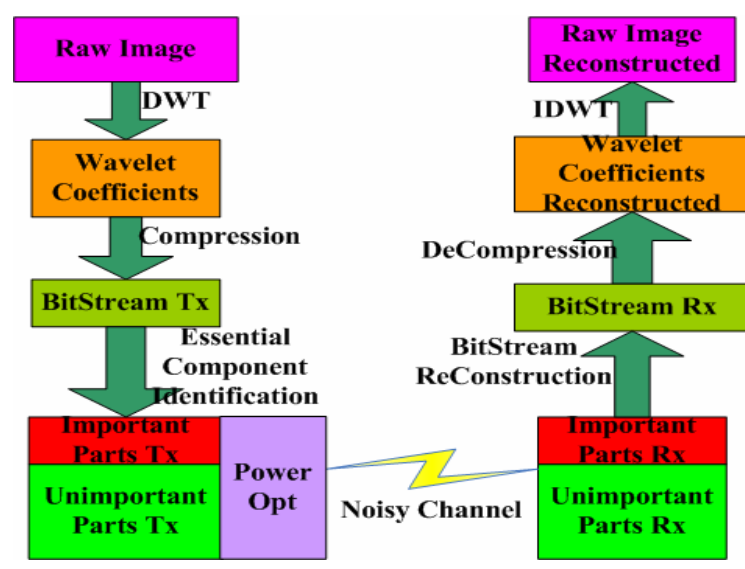

Fig. 3: Image Component Transmission Data Flow in WSN

In wavelet image compression algorithms, wavelet coefficients are compressed into embedded bitstreams after DWT is applied onto raw image. The bitstreams processed after essential components identification take the form shown in Figure 2, in which all the 
components are identified, and the corresponding importance is to be exploited during transmission. Unequal importance transmissions are applied to those bitstreams over wireless channel, with desirable transmission power supplied. At the sink node, bitstreams are reconstructed and decompressed into wavelet coefficients, and raw image is reconstructed by applying inverse DWT.

Instead of transmitting the whole compressed image uniformly with equal importance, different desirable BER requirements are applied to different compression component. To exploit unequal importance transmission, a component segmentation table is created in the form of Table 1 at application layer. Each component in the embedded bitstreams has an entry in this segmentation table, followed by a desirable BER requirement and offset position in the bitstream. The desirable transmission power is now shown in this segmentation since it can be calculated from desirable BER requirements, with the channel attenuation factor, symbol rate and modulation scheme ${ }^{[7-8]}$.

Table 1: Component Based Segmentation Table. The Offset byte is used for lower MAC-PHY layer to identify each component. Offset bytes include layer overhead from application layer through network layer to MAC-PHY

\begin{tabular}{lll}
\hline \multicolumn{1}{c}{ Component } & Desirable BER & Offset Byte \\
\hline H0p & BER0p & O0p \\
H0v & BER0v & O0v \\
$\ldots \ldots$. & $\ldots \ldots$ & $\ldots \ldots$ \\
H(n-1)p & BER(n-1)p & O(n-1)p \\
H(n--1)v & BER(n-1)v & O(n-1)p \\
\hline
\end{tabular}

The desirable BER requirement is assigned according to the importance of that component. Generally, components containing structure information has higher desirable BER requirement than components containing magnitude information. For all those components containing structure information, they have a descending importance sequence if the wavelet resolution level increases. This is also applicable for unimportant components containing magnitude information. Such concept is based on the fact that lower resolution of image data is more critical than high resolution to assure basic transmission quality requirement in WSN. The following equations show this general guideline of desirable BER sequence:

$$
\begin{aligned}
& B E R_{i, p} \leq B E R_{i, v}, 0 \leq i \leq n-1 \\
& B E R_{i, p} \leq B E R_{j, p}, 0 \leq i \leq j \leq n-1 \\
& B R_{i, v} \leq B E R_{j, v}, 0 \leq i \leq j \leq n-1
\end{aligned}
$$

Figure 4 shows the ICT process. Each component is packed by MAC-PHY with the knowledge of desirable BER requirement and the offset of that component. The components in segmentation table are in the same order as the embedded bitstream, starting with HOp and ending with Hnv. Therefore, MAC-PHY can identify each component for transmission by reading the offset byte in the segmentation table. Components in the output bitstream are scanned and transmitted in sequence, at the corresponding variable BER and desirable transmission power, instead of being transmitted with uniform BER and uniform power. The transmission power must be dynamically adjustable according to the desirable BER requirement, current channel attenuation factor and modulation scheme parameters. In this paper we focus on single rate transmission, and the modulation scheme parameters are not variables of desirable transmission power. Desirable transmission power can be determined through Equation (16) or (17), given desirable BER and channel attenuation factor. Channel attenuation factor can be acquired via RTS-CTS handshake, and detailed description can be referenced in $^{[8]}$.

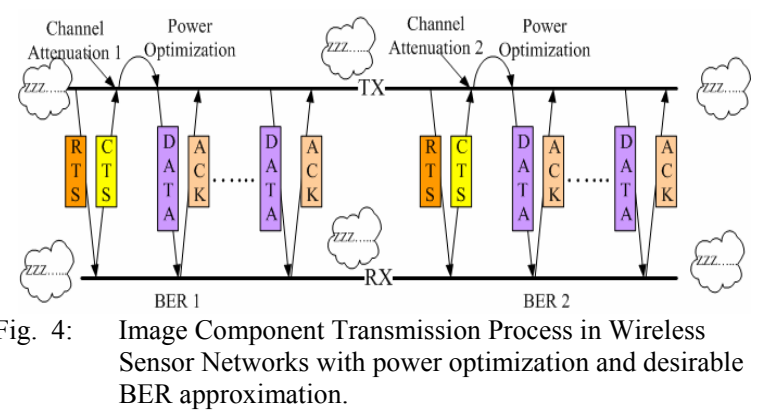

Instead of treating the compressed image bit stream by a universal desirable BER requirement, the ICT scheme applies different desirable BER requirements to different image components. Given the same other factors, the energy consumption of transmitting each image component is only related to component size, desirable BER requirement and the actual transmission rate. The number of components and the size of each component are determined by the compression process of wavelet coefficients.

\section{SIMULATION}

In this section, we show the effectiveness of ICT scheme in achieving energy efficiency while enhancing image transmission quality. The digital image is transmitted between sensor nodes, by applying uniform protection and transmission $\left.{ }^{[1,} 10\right]$, layer oriented 
transmission ${ }^{[2,9]}$, and the proposed component based transmission schemes. Performance data such as energy consumption and the reconstructed image PSNR values are recorded and compared.

In our simulation, we employ T-MAC ${ }^{[11]}$ as MAC protocol. The evaluation metrics are defined as the following. For T-MAC data packets in TinyOS ${ }^{[12]}$, the MAC header is 11 bytes and the payload is 36 bytes. For the control packet such as ACK, the length is 13 bytes. RTS and CTS packets are both 15 bytes. The TA value of T-MAC used in the simulation is the same as the time of transmitting a RTS packet. Preamble length in physical layer is 18 bytes ${ }^{[13]}$. The receive power is fixed to $0.01 \mathrm{~mW}$, and the sleep power is $0.00002 \mathrm{~mW}$, while idle power is $0.009 \mathrm{~mW}$, according to the same parameters scales in ${ }^{[14]}$. Channel attenuation factor $A$ is $-90 \mathrm{db}$. The noise power density $N_{0}$ is the product of Boltzmann constant $1.38 \times 10^{-23}$ and equivalent noise temperature $T n$. Assume the noise temperature is normal room temperature $290 \mathrm{~K}$, and the noise power density value $4 \times 10^{-21} \mathrm{~J} / \mathrm{Hz}$. Figure 5 shows the relationship between the packet level energy consumption and the desirable BER requirement value.

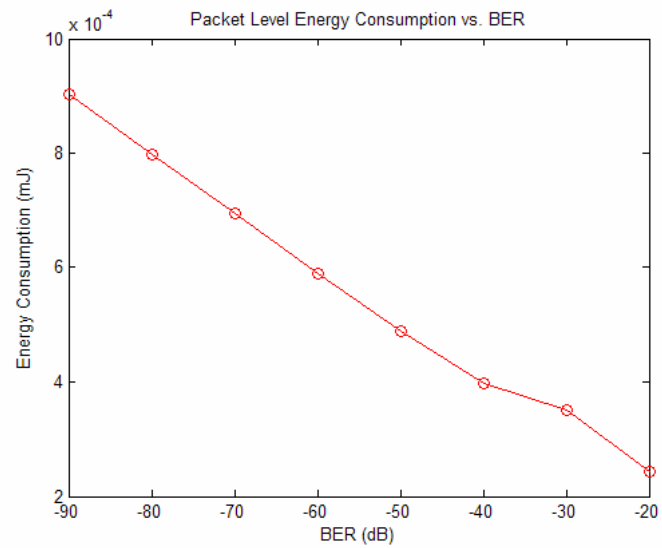

Fig. 5: Average Energy Consumption per Packet with desirable BER requirements.

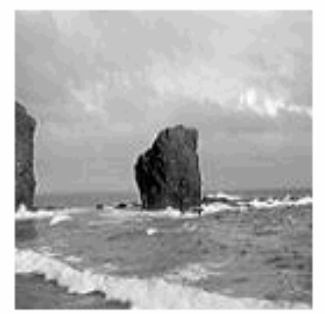

Fig. 6: Original image with 8 bits per pixel and $128 * 128$ pixels transmitted between sensor nodes.

Figure 6 shows an original image to be compressed and transmitted between sensor nodes. As proposed in
ICT scheme, each component in the wavelet compressed image bit stream is assigned a corresponding desirable BER requirement value shown in Table 2. All the components in the first column of Table 2 are grouped as a vector corresponding to the original image. Three scenarios including low noise (S1), medium noise (S2) and strong noise (S3) channel environments are simulated and studied. In each scenario, three transmission approaches - uniform approach, layered approach, component (i.e., ICT) based approach - are analyzed. Each approach has three desirable BER vectors in those scenarios in Table 2.

Table 2: Desirable BER requirements vector assignment for the component vector in compressed image stream. Each column corresponds to a desirable BER requirement vector. 3 scenarios corresponding to different channel noise levels are studied for all the three approaches

\begin{tabular}{|c|c|c|c|c|c|c|c|c|c|}
\hline Com & \multicolumn{4}{|c|}{ Uniform BER Vector } & \multicolumn{2}{|c|}{$\begin{array}{c}\text { Layered BER } \\
\text { Vector }\end{array}$} & \multicolumn{3}{|c|}{$\begin{array}{c}\text { Component BER } \\
\text { Vector }\end{array}$} \\
\hline Scen & S1 & S2 & S3 & S1 & S2 & S3 & S1 & S2 & S3 \\
\hline H0p & $1 \mathrm{e}-3$ & $5 e-3$ & $1 \mathrm{e}-2$ & $1 \mathrm{e}-4$ & $1 \mathrm{e}-3$ & $1 \mathrm{e}-3$ & $1 \mathrm{e}-4$ & $1 \mathrm{e}-3$ & $4 \mathrm{e}-3$ \\
\hline Hlp & $1 e-3$ & $5 e-3$ & $1 \mathrm{e}-2$ & $1 \mathrm{e}-4$ & $1 e-3$ & $1 e-3$ & $1 \mathrm{e}-4$ & $1 e-3$ & $4 e-3$ \\
\hline $\mathrm{H} 2 \mathrm{p}$ & $1 e-3$ & $5 e-3$ & $1 \mathrm{e}-2$ & $1 \mathrm{e}-4$ & $1 \mathrm{e}-3$ & $1 e-3$ & $1 \mathrm{e}-4$ & $2 e-3$ & $4 e-3$ \\
\hline $\mathrm{H} 3 \mathrm{p}$ & $1 e-3$ & $5 e-3$ & $1 \mathrm{e}-2$ & $1 \mathrm{e}-3$ & $2 e-3$ & $1 \mathrm{e}-2$ & $1 \mathrm{e}-4$ & $2 e-3$ & $4 e-3$ \\
\hline $\mathrm{H} 4 \mathrm{p}$ & $1 e-3$ & $5 e-3$ & $1 \mathrm{e}-2$ & $1 \mathrm{e}-3$ & $4 e-3$ & $1 \mathrm{e}-2$ & $1 \mathrm{e}-4$ & $3 e-3$ & $4 e-3$ \\
\hline H5p & $1 e-3$ & $5 e-3$ & $1 \mathrm{e}-2$ & $1 \mathrm{e}-3$ & $5 e-3$ & $1 \mathrm{e}-2$ & $1 \mathrm{e}-4$ & $3 e-3$ & $5 e-3$ \\
\hline H6p & $1 e-3$ & $5 e-3$ & $1 \mathrm{e}-2$ & $1 \mathrm{e}-3$ & $5 e-3$ & $1 \mathrm{e}-2$ & $1 e-3$ & $3 e-3$ & $6 e-3$ \\
\hline $\mathrm{H} 7 \mathrm{p}$ & $1 e-3$ & $5 e-3$ & $1 \mathrm{e}-2$ & $1 \mathrm{e}-3$ & $5 e-3$ & $1 \mathrm{e}-2$ & $1 e-3$ & $5 e-3$ & $7 e-3$ \\
\hline $\mathrm{H} 8 \mathrm{p}$ & $1 e-3$ & $5 e-3$ & $1 \mathrm{e}-2$ & $1 \mathrm{e}-3$ & $5 e-3$ & $1 \mathrm{e}-2$ & $1 e-3$ & $5 e-3$ & $8 e-3$ \\
\hline H9p & $1 e-3$ & $5 e-3$ & $1 \mathrm{e}-2$ & $1 \mathrm{e}-3$ & $5 e-3$ & $1 \mathrm{e}-2$ & $1 e-3$ & $5 e-3$ & $8 e-3$ \\
\hline H10 & $1 e-3$ & $5 e-3$ & $1 \mathrm{e}-2$ & $1 e-3$ & $5 e-3$ & $1 \mathrm{e}-2$ & $1 e-3$ & $5 e-3$ & $8 e-3$ \\
\hline $\begin{array}{l}\mathrm{p} \\
\mathrm{H} 11\end{array}$ & $1 e-3$ & $5 e-3$ & $1 \mathrm{e}-2$ & $2 \mathrm{e}-3$ & $5 e-3$ & $2 \mathrm{e}-2$ & $1 \mathrm{e}-3$ & $5 \mathrm{e}-3$ & $8 e-3$ \\
\hline $\mathrm{p}$ & & & & & & & & & \\
\hline $\mathrm{H} 0 \mathrm{v}$ & $1 e-3$ & $5 e-3$ & $1 \mathrm{e}-2$ & 1e-4 & $1 e-3$ & $1 e-3$ & $1 e-3$ & $6 e-3$ & $8 \mathrm{e}-2$ \\
\hline $\mathrm{H} 1 \mathrm{v}$ & $1 \mathrm{e}-3$ & $5 e-3$ & $1 \mathrm{e}-2$ & $1 \mathrm{e}-4$ & $1 \mathrm{e}-3$ & $1 e-3$ & $1 \mathrm{e}-3$ & $6 e-3$ & $8 \mathrm{e}-2$ \\
\hline $\mathrm{H} 2 \mathrm{v}$ & $1 e-3$ & $5 e-3$ & $1 \mathrm{e}-2$ & $1 \mathrm{e}-4$ & $1 e-3$ & $1 e-3$ & $1 \mathrm{e}-3$ & $7 e-3$ & $1 \mathrm{e}-1$ \\
\hline $\mathrm{H} 3 \mathrm{v}$ & $1 \mathrm{e}-3$ & $5 e-3$ & $1 \mathrm{e}-2$ & $1 \mathrm{e}-3$ & $2 e-3$ & $1 \mathrm{e}-2$ & $1 \mathrm{e}-3$ & $8 \mathrm{e}-3$ & $1 \mathrm{e}-1$ \\
\hline $\mathrm{H} 4 \mathrm{v}$ & $1 \mathrm{e}-3$ & $5 e-3$ & $1 \mathrm{e}-2$ & $1 \mathrm{e}-3$ & $4 e-3$ & $1 \mathrm{e}-2$ & $1 \mathrm{e}-3$ & $8 \mathrm{e}-3$ & $1 \mathrm{e}-1$ \\
\hline $\mathrm{H} 5 \mathrm{v}$ & $1 e-3$ & $5 e-3$ & $1 \mathrm{e}-2$ & $1 e-3$ & $5 e-3$ & $1 \mathrm{e}-2$ & $1 \mathrm{e}-3$ & $9 e-3$ & $1 \mathrm{e}-1$ \\
\hline $\mathrm{H} 6 \mathrm{v}$ & $1 \mathrm{e}-3$ & $5 e-3$ & $1 \mathrm{e}-2$ & $1 \mathrm{e}-3$ & $5 e-3$ & $1 \mathrm{e}-2$ & $1 \mathrm{e}-2$ & $9 \mathrm{e}-3$ & $1 \mathrm{e}-1$ \\
\hline $\mathrm{H} 7 \mathrm{v}$ & $1 \mathrm{e}-3$ & $5 e-3$ & $1 \mathrm{e}-2$ & $1 \mathrm{e}-3$ & $5 e-3$ & $1 \mathrm{e}-2$ & $1 \mathrm{e}-2$ & $9 e-3$ & $1 \mathrm{e}-1$ \\
\hline $\mathrm{H} 8 \mathrm{v}$ & $1 e-3$ & $5 e-3$ & $1 \mathrm{e}-2$ & $1 e-3$ & $5 e-3$ & $1 \mathrm{e}-2$ & $1 \mathrm{e}-2$ & $1 e-2$ & $1 \mathrm{e}-1$ \\
\hline $\mathrm{H} 9 \mathrm{v}$ & $1 \mathrm{e}-3$ & $5 e-3$ & $1 \mathrm{e}-2$ & $1 \mathrm{e}-3$ & $5 e-3$ & $1 \mathrm{e}-2$ & $1 \mathrm{e}-2$ & $1 \mathrm{e}-2$ & $1 \mathrm{e}-1$ \\
\hline $\mathrm{H} 10$ & $1 \mathrm{e}-3$ & $5 e-3$ & $1 \mathrm{e}-2$ & $1 e-3$ & $5 e-3$ & $1 \mathrm{e}-2$ & $1 \mathrm{e}-2$ & $1 \mathrm{e}-2$ & $1 \mathrm{e}-1$ \\
\hline
\end{tabular}

Figure 7,8 and 9 show the image transmission quality comparisons among different approaches for low noise scenario S1, medium noise S2 and strong noise $\mathrm{S} 3$, respectively. In each, the three approaches are compared with performances in terms of the image transmission quality and energy consumption results. The reconstructed images with the proposed component based transmission approach (i.e., ICT approach in index (c) in those three figures) achieve the best image transmission quality, while maintain the least energy consumption. With ICT approach, more effort is put on those more important components (i.e., $p$ data), which leads to higher image quality; while less effort is put on those unimportant components (i.e., $v$ data), which reduces energy consumption. The increasing of image transmission quality by putting more effort on essential components incurs transmission energy overhead; however, as long as the decreased energy consumption 
due to less effort put on unimportant components overcome that overhead, image quality improvement and energy efficiency are both achieved.

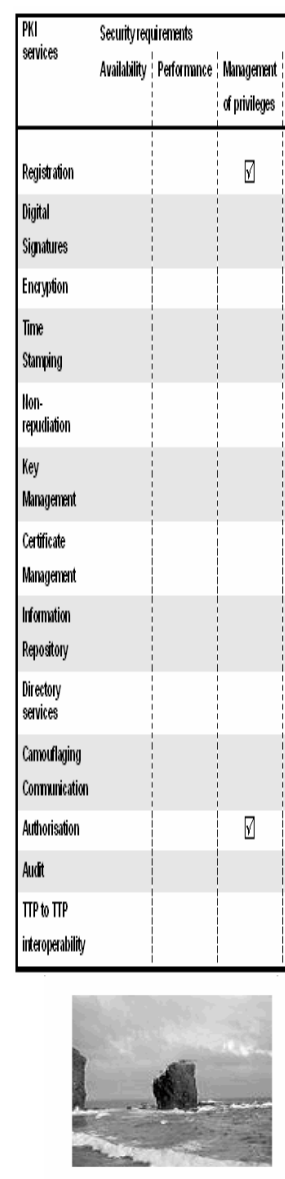

(a)

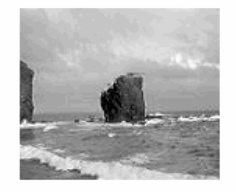

(b)

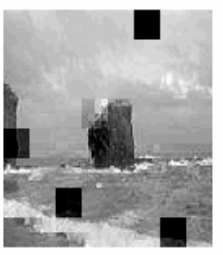

(a)

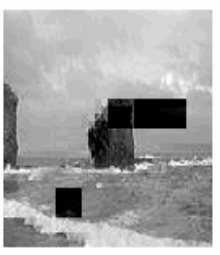

(b)

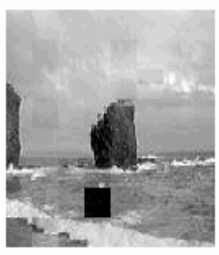

(c)
Fig. 9: Comparison of reconstructed images for strong noise scenario S3. Black blocks in these figures are the results of transmission errors on important components ( $p$ data), which lead to errors with regard to the pixel positions. Translucent blocks are due to the errors with regard to the pixel values. (a) Uniform approach, PSNR $=16.224 \mathrm{~dB}$, energy consumption $E=0.60121 \mathrm{~mJ}$; (b) Layered approach, PSNR $=16.6842 \mathrm{~dB}, \mathrm{E}=0.5711 \mathrm{~mJ}$; (c) Component approach, PSNR $=22.7599 \mathrm{~dB} . \mathrm{E}=0.5602 \mathrm{~mJ}$.

Figure 10 shows the image quality at different channel conditions, for all the three approaches. With all the other factors being the same, the proposed ICT approach has higher image transmission quality than uniform approach and layered approach.

Figure 11 shows the performance relationship between energy consumption and image quality. By exploring the unequal importance nature of various components in the compressed image data, the proposed ICT approach is effective to reduce energy consumption while enhancing image quality.

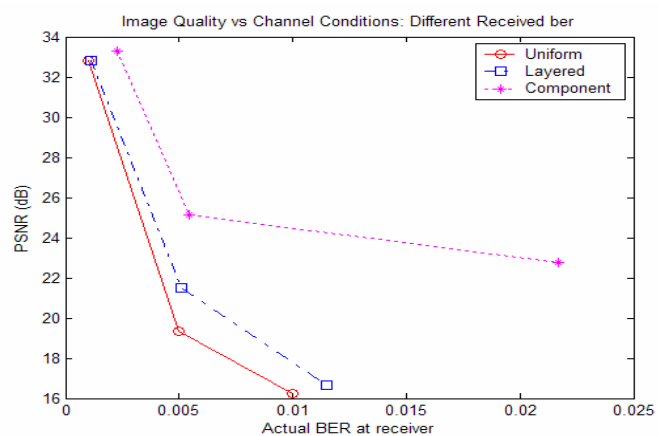

Fig. 10: Reconstructed image quality at different channel conditions.

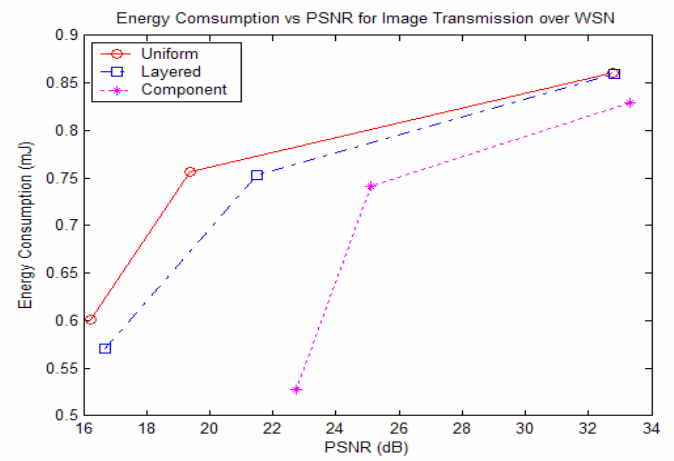

Fig. 11: Energy consumptions at different image quality level 


\section{CONCLUSION}

In image sensor based WSN, visual information are obtained via a large number of medium-quality lowcost image sensor nodes. In such a system, digital images are transmitted in noisy channels from multiple source sensors under resource constraints, and the visual field information is collected through large number of medium- or low-quality images captured by the source sensors. One of the critical challenges to such system design is the tradeoff of image transmission quality with energy efficiency.

In this paper, we proposed a novel Image Component Transmission approach to enhance image transmission quality in WSN. Our contribution lies in two aspects - (1) the identification of components with various importance levels in compression bit streams and (2) a practical proposal for different transmission strategies adaptive to different components. The compressed image bit stream is componentized and differently transmitted. The unequal importance of components for image reconstruction is explored. The important components which contain bit structure information with regard to the position of image pixels are more reliably protected and transmitted, while unimportant part is relatively less protected. Simulations results show that the proposed ICT approach increases the image transmission quality considerably on noisy channels, while at the same time it achieves the energy efficiency.

\section{REFERENCES}

1. L.Ferrigno, S.Marano, V.Paciello, A.Pietrosanto, 2005. Balancing computational and transmission power consumption in wireless image sensor networks. IEEE International Conference on Virtual Environments, Human-Computer Interfaces and Measurement Systems, 1 (1): 61- 66.

2. W.Yu, Z.Sahinoglu, A.Vetro, 2004. Energy efficient JPEG 2000 image transmission over wireless sensor networks. IEEE Global Telecommunications Conference., 5 (1): 2738 2743.

3. J.M.Shapiro, 1993. Embedded image coding using zerotrees of wavelet coefficients. IEEE Transactions on Signal Processing., 41 (12): 3445 3462.
4. A.Said, W.A.Pearlman, 1996. A new, fast, and efficient image codec based on set partitioning in hierarchical trees. IEEE Transactions on Circuits and Systems for Video Technology., 6 (3): 243 250.

5. D.Taubman, 2000. High performance scalable image compression with EBCOT. IEEE Transactions on Image Processing., 9 (7): 1158 1170.

6. H.Cheng, X.Li, 2000. Partial encryption of compressed images and videos. IEEE Transactions on Signal Processing., 48 (8): 2439 - 2451.

7. W. Wang, D. Peng, H.Wang, H. Sharif, 2006 . Study of an Energy Efficient Multi Rate Scheme for Wireless Sensor Network MAC. In: The 2nd ACM International Workshop on QoS and Security for Wireless Networks, pp.51 - 54.

8. W.Wang, D.Peng, H.Wang, H.Sharif, T.Wysocki, B.Wysocki, 2006. An Energy Efficient MAC-PHY Approach to Support Distributed Source Coding in Wireless Sensor Network. In: The 5th Workshop on the Internet, Telecommunications and Signal Processing.

9. M.Wu, C.Chen, 2003. Multiple bitstream image transmission over wireless sensor networks. In: Proceedings of IEEE Sensors, pp.727-731.

10. C.Chiasserini, E.Magli, 2002. Energy consumption and image quality in wireless video-surveillance networks. In: The 13th IEEE International Symposium on Personal, Indoor and Mobile Radio Communications. pp.2357 - 2361.

11. T. van Dam, K. Langendoen, 2003. An adaptive energy efficient MAC protocol for wireless sensor networks. In: Proceedings of the First ACM Conference on Embedded Networked Sensor Systems. Pp. 171 - 180.

12. http://www.tinyos.net

13. I. Rhee, A. Warrier, M. Aia, J. Min, 2005. Z-MAC: a hybrid MAC for wireless sensor networks. In: Proceedings of the 3 rd international conference on Embedded networked sensor systems, pp. $90-101$.

14. J. Polastre, J. Hill, and D. Culler, 2004. Versatile low power media access for wireless sensor networks. In: Proceedings of the Second ACM Conference on Embedded Networked Sensor Systems. pp.95 - 107. 